\title{
Numerical Viscosity and the Entropy Condition for Conservative Difference Schemes*
}

\author{
By Eitan Tadmor**
}

\begin{abstract}
Consider a scalar, nonlinear conservative difference scheme satisfying the entropy condition. It is shown that difference schemes containing more numerical viscosity will necessarily converge to the unique, physically relevant weak solution of the approximated conservative equation. In particular, entropy satisfying convergence follows for $E$ schemes - those containing more numerical viscosity than Godunov's scheme.
\end{abstract}

1. Introduction. There is a close relation between the concepts of entropy and viscosity, associated with systems of conservation laws. It is well known, for example, that vanishing viscosity weak solutions for such systems must satisfy the entropy inequality across their discontinuities, and that the converse holds, at least in the small (in the large for scalar problems); both are used to identify the so-called "physically relevant" solution of such systems, e.g., [7].

In this paper we amplify a certain aspect of this relation, with regard to conservative difference schemes

$$
\begin{aligned}
v_{\nu}(t+k)= & v_{\nu}(t) \\
& -\lambda\left[h\left(v_{\nu-p+1}(t), \ldots, v_{\nu+p}(t)\right)-h\left(v_{\nu-p}(t), \ldots, v_{\nu+p-1}(t)\right)\right]
\end{aligned}
$$

serving as consistent approximations to the scalar conservation law

$$
\frac{\partial u}{\partial t}(x, t)+\frac{\partial f}{\partial x}(u(x, t))=0
$$

To make our point, consider a difference scheme which is known to satisfy the entropy inequality; roughly speaking, this should indicate according to the above, the existence of a certain amount of numerical viscosity present in such a scheme. It is therefore plausible to assert that other schemes, containing more numerical viscosity, will also have to satisfy the entropy inequality. After putting these terms in a more precise framework, we show the validity of the above assertion subject to the technical assumptions listed below. Thus, we prove the entropy inequality by means of comparison.

Received June 20, 1983; revised February 17, 1984.

1980 Mathematics Subject Classification. Primary 65P05, 35L65.

*Research was supported by the National Aeronautics and Space Administration under NASA Contract No. NAS1-17070 while the author was in residence at ICASE, NASA Langley Research Center, Hampton, VA 23665.

${ }^{* *}$ Current address. School of Mathematical Sciences, Tel-Aviv University, Tel-Aviv 69978, Israel. 
In [12], Osher introduced, for the method of lines, a class of $E$ schemes which were shown to converge to the physically relevant solution. Making use of the terminology just introduced, the so-called $E$ schemes can be identified as exactly those having no less numerical viscosity than that of Godunov. Since the latter is known to satisfy the entropy inequality, we are able to extend Osher's ideas to the fully discrete case, as a special case of the above assertion. This is carried out in Section 5, paving the way for the proof of the more general assertion in Section 6. Prior to that, we give in Sections 3 and 4 a brief discussion of the entropy inequality in relation to the all important Godunov and Lax-Friedrichs schemes.

2. Preliminaries. We consider difference schemes

$$
v_{\nu}(t+k)=H\left(v_{\nu-p}(t), \ldots, v_{\nu+p}(t) ; f, \lambda\right)
$$

which admit a conservative form

$$
\begin{aligned}
& H\left(v_{\nu-p}, \ldots, v_{\nu+p} ; f, \lambda\right) \\
& \quad=v_{\nu}-\lambda\left[h\left(v_{\nu-p+1}, \ldots, v_{\nu+p}\right)-h\left(v_{\nu-p}, \ldots, v_{\nu+p-1}\right)\right],
\end{aligned}
$$

and are serving as consistent approximations to the scalar conservation law

$$
\frac{\partial u}{\partial t}(x, t)+\frac{\partial f}{\partial x}(u(x, t))=0
$$

Here, $v_{\nu}(t) \equiv v\left(x_{\nu}, t\right)$ denotes the approximation value at the gridpoint $\left(x_{\nu} \equiv \nu \Delta x, t\right)$, $k$ and $\Delta x$ are respectively, the temporal and spatial mesh size such that the mesh ratio $\lambda \equiv k / \Delta x$ is being kept fixed, and $p$, a natural number. Finally, $h_{\nu+1 / 2} \equiv$ $h\left(v_{\nu-p+1}, \ldots, v_{\nu+p}\right)$ is the Lipschitz continuous numerical flux consistent with the differential one, $h(w, w, \ldots, w)=f(w)$; for the sake of simplifying the notations, its possible dependence on $f$ and $\lambda$ is suppressed.

We begin by putting the scheme (2.1) in an increment form: using the difference operator $\Delta w_{\nu+1 / 2} \equiv w_{\nu+1}-w_{\nu}$, we set for $v_{\nu+1} \neq v_{\nu}$

$$
\begin{gathered}
C_{\nu+1 / 2}^{+}=\lambda \frac{f_{\nu}-h_{\nu+1 / 2}}{\Delta v_{\nu+1 / 2}}, \\
C_{\nu+1 / 2}^{-}=\lambda \frac{f_{\nu+1}-h_{\nu+1 / 2}}{\Delta v_{\nu+1 / 2}} ;
\end{gathered}
$$

adding and subtracting $f_{\nu}$ to the RHS of (2.1b) and making use of (2.3), (2.1a) reads

$$
v_{\nu}(t+k)=v_{\nu}(t)+C_{\nu+1 / 2}^{+} \Delta v_{\nu+1 / 2}(t)-C_{\nu-1 / 2}^{-} \Delta v_{\nu-1 / 2}(t) .
$$

Next, we denote

$$
Q_{\nu+1 / 2} \equiv C_{\nu+1 / 2}^{+}+C_{\nu+1 / 2}^{-}=\lambda \frac{f_{\nu}+f_{\nu+1}-2 h_{\nu+1 / 2}}{\Delta v_{\nu+1 / 2}}
$$

Noting the identity

$$
C_{\nu+1 / 2}^{-}-C_{\nu+1 / 2}^{+}=\lambda \frac{\Delta f_{\nu+1 / 2}}{\Delta v_{\nu+1 / 2}}
$$

the incremental coefficients $C_{\nu+1 / 2}^{ \pm}$equal

$$
C_{\nu+1 / 2}^{ \pm}=\frac{1}{2}\left(Q_{\nu+1 / 2} \mp \lambda \frac{\Delta f_{\nu+1 / 2}}{\Delta v_{\nu+1 / 2}}\right) \text {. }
$$


Inserting this into (2.4), our scheme then assumes the form

$$
\begin{aligned}
v_{\nu}(t+k)= & v_{\nu}(t)-\frac{\lambda}{2}\left[f\left(v_{\nu+1}(t)\right)-f\left(v_{\nu-1}(t)\right)\right] \\
& +\frac{1}{2}\left[\Delta\left(Q_{\nu-1 / 2} \Delta v_{\nu-1 / 2}(t)\right)\right]
\end{aligned}
$$

which reveals the role $Q$ plays as the numerical viscosity coefficient. We will therefore use $Q$ as a measurement of the amount of viscosity present in such a scheme.

Remark. In the case of 3-point schemes, $p=1$, this measure of viscosity is rather general in the sense that such schemes are completely determined by their coefficient of numerical viscosity, e.g., [10]. We do not claim such generality for $(2 p+1)$-point schemes $p>1$ : this definition of numerical viscosity is in fact 3-point oriented, as we shall see in a more precise form later on.

Let $\operatorname{TV}[v(t)] \equiv \sum_{\nu}\left|v_{\nu+1}(t)-v_{\nu}(t)\right|$ denote the total variation of the computed solution at time $t$; we then have the following

LEMMA 2.1. The scheme (2.1) is total variation nonincreasing provided its numerical viscosity coefficient, $Q_{\nu+1 / 2}$, satisfies

$$
\lambda\left|\frac{\Delta f_{\nu+1 / 2}}{\Delta v_{\nu+1 / 2}}\right| \leqslant Q_{\nu+1 / 2} \leqslant 1 .
$$

Proof. The inequalities (2.8), expressed in terms of the incremental coefficients in (2.6), are translated into

$$
C_{\nu+1 / 2}^{+} \geqslant 0, \quad C_{\nu+1 / 2}^{-} \geqslant 0, \quad 1-C_{\nu+1 / 2}^{+}-C_{\nu+1 / 2}^{-} \geqslant 0 .
$$

A straightforward calculation, based on the incremental form (2.4) and the inequalities (2.9), shows the nonincrease in total variation, $\operatorname{TV}[v(t+k)] \leqslant \operatorname{TV}[v(t)]$, see [5].

Lemma 2.1 implies, in particular, the convergence of the scheme (2.1), provided its numerical viscosity coefficient meets the requirement (2.8): one can select a boundedly a.e. converging subsequence, $v_{\nu}\left(t ; \Delta x^{\prime}\right)$, such that its limit

$$
v(x, t)=\lim _{x=\nu \Delta x^{\prime}, \Delta x^{\prime} \rightarrow 0} v_{\nu}\left(t ; \Delta x^{\prime}\right)
$$

satisfies (2.2) in the weak sense, e.g., [1], [3], [9].*** Weak solutions of (2.2) however, are not necessarily unique. The lower bound on the LHS of $(2.8)$ requiring that much of viscosity for convergence to a limit weak solution, does not guarantee this weak solution to be the physically relevant one: it is well known, for example, that the 3-point Courant-Isaacson-Rees scheme where $Q_{\nu+1 / 2}=\lambda\left|\Delta f_{\nu+1 / 2} / \Delta v_{\nu+1 / 2}\right|$, may admit limit weak solutions violating the physically relevant entropy condition, e.g., [5], [12]; thus, a greater amount of viscosity is required for the entropy condition to hold. In the next section we discuss Godunov's scheme which turns out to play a central role in determining that additional required amount.

We note in passing, the fundamentally different role played by the upper bound on the numerical viscosity, appearing on the RHS of (2.8). It is related to the hyperbolic nature of the approximated equation (2.2), as it amounts to the CFL-like condition,

\footnotetext{
***We consider compactly supported initial data; a further $L^{\infty}$ bound, derived below, is required for the more general initial data in $L^{1} \cap L^{\infty} \cap B V$.
} 
see (2.5),

$$
\lambda\left|\left(f\left(v_{\nu}\right)-h_{\nu+1 / 2}\right)+\left(f\left(v_{\nu+1}\right)-h_{\nu+1 / 2}\right)\right| \leqslant\left|v_{\nu+1}-v_{\nu}\right|
$$

which usually results in placing a limitation on the mesh ratio, $\lambda$, being used (recall that $h(\cdots)$ may depend on $\lambda$ as well). A stricter CFL condition of this type was introduced in [9]. In particular, the numerical flux of a difference scheme satisfying (2.10) admits the consistency relation

$$
h\left(v_{\nu-p+1}, \ldots, v_{\nu-1}, w, w, v_{\nu+2}, \ldots, v_{\nu+p}\right)=f(w) .
$$

Such essentially 3-point schemes include, beside the standard 3-point schemes, several of the recently constructed second-order accurate converging schemes, e.g., [5], [8].

Finally, we would like to point out that by halving the CFL number, one obtains a maximum principle; that is,

LEMMA 2.2. Consider the scheme (2.1) with a numerical viscosity coefficient, $Q_{\nu+1 / 2}$, satisfying

$$
\lambda\left|\frac{\Delta f_{\nu+1 / 2}}{\Delta v_{\nu+1 / 2}}\right| \leqslant Q_{\nu+1 / 2} \leqslant \frac{1}{2} .
$$

Then, the following maximum principle

$$
\inf _{\mu}\left[v_{\mu}(t)\right] \leqslant v_{\nu}(t+k) \leqslant \sup _{\mu}\left[v_{\mu}(t)\right]
$$

holds.

Proof. The incremental coefficients in (2.6) do not exceed a value of

$$
0 \leqslant C_{\nu+1 / 2}^{ \pm}=\frac{1}{2}\left(Q_{\nu+1 / 2} \mp \lambda \frac{\Delta f_{\nu+1 / 2}}{\Delta v_{\nu+1 / 2}}\right) \leqslant \frac{1}{2} \cdot 2 Q_{\nu+1 / 2} \leqslant \frac{1}{2} \text {. }
$$

Making use of the incremental form of the scheme, see (2.4),

$$
v_{\nu}(t+k)=C_{\nu+1 / 2}^{+} v_{\nu+1}(t)+\left(1-C_{\nu+1 / 2}^{+}-C_{\nu-1 / 2}^{-}\right) v_{\nu}(t)+C_{\nu-1 / 2}^{-} v_{\nu-1}(t),
$$

and noting the convexity of the combination on the RHS, (2.13) follows.

3. The Entropy Condition and Godunov's Scheme. The building block in Godunov's scheme, [4], is the solution of the Riemann problem. Let $u^{R}\left(x / t ; u_{\text {left }}, u_{\text {right }}\right)$ denote the similarity solution of the Riemann problem (2.2) subject to initial condition

$$
u(x, t=0)=\frac{1-\operatorname{sgn}(x)}{2} u_{\text {left }}+\frac{1+\operatorname{sgn}(x)}{2} u_{\text {right }} .
$$

Godunov's scheme is determined by

$$
v_{\nu}(t+k)=H^{G}\left(v_{\nu-1}, v_{\nu}, v_{\nu+1}\right) \equiv \frac{v_{\nu-1 / 2}^{-}+v_{\nu+1 / 2}^{+}}{2}
$$

where

$$
\begin{aligned}
& v_{\nu-1 / 2}^{-} \equiv v^{-}\left(v_{\nu-1}, v_{\nu}\right)=\frac{1}{\Delta x / 2} \int_{0}^{\Delta x / 2} u^{R}\left(x / k ; v_{\nu-1}, v_{\nu}\right) d x \\
& v_{\nu+1 / 2}^{+} \equiv v^{+}\left(v_{\nu}, v_{\nu+1}\right)=\frac{1}{\Delta x / 2} \int_{-\Delta x / 2}^{0} u^{R}\left(x / k ; v_{\nu}, v_{\nu+1}\right) d x .
\end{aligned}
$$


Assume the CFL condition

$$
\lambda \cdot \max _{u}|a(u)| \leqslant \frac{1}{2}, \quad a(u) \equiv \dot{f}(u)
$$

holds. The RHS of $(3.1 \mathrm{~b}-\mathrm{c})$ can be evaluated from the integral form of $(2.2)$, see Figure 3-1, giving

$$
\begin{aligned}
& v_{\nu-1 / 2}^{-}=v_{\nu}-2 \lambda\left(f_{\nu}-h_{\nu-1 / 2}^{G}\right), \\
& v_{\nu+1 / 2}^{+}=v_{\nu}+2 \lambda\left(f_{\nu}-h_{\nu+1 / 2}^{G}\right),
\end{aligned}
$$

where

$$
h_{\nu+1 / 2}^{G}=h^{G}\left(v_{\nu}, v_{\nu+1}\right)=f\left(u^{R}\left(0^{+} ; v_{\nu}, v_{\nu+1}\right)\right)
$$

stands for the numerical flux of Godunov's scheme: indeed, by averaging $(3.2 \mathrm{~b}-\mathrm{c})$, (3.1a) takes the desired conservative form

$$
v_{\nu}(t+k)=v_{\nu}-\lambda\left(h_{\nu+1 / 2}^{G}-h_{\nu-1 / 2}^{G}\right) .
$$

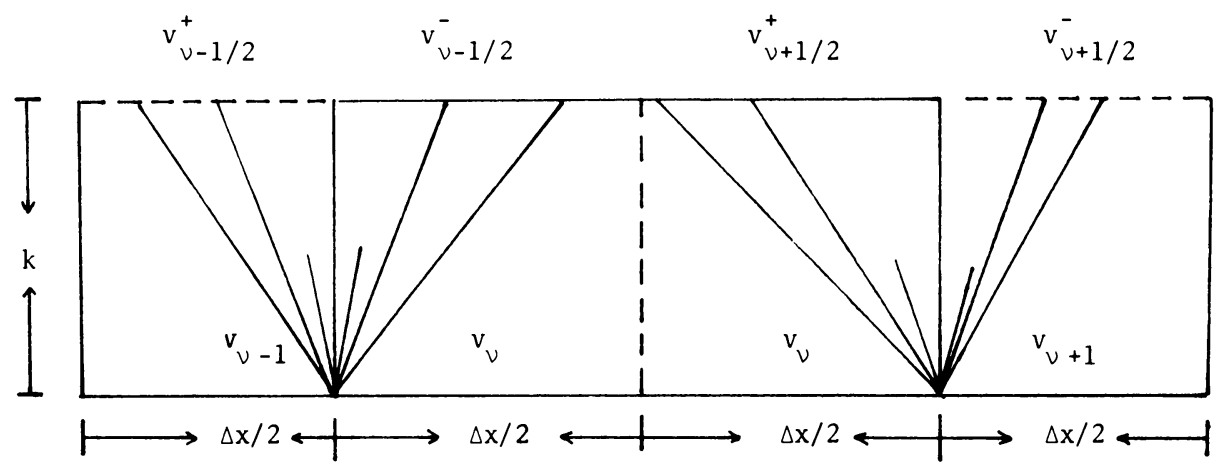

FIGURE 3-1

Consider a pair of scalar functions $(U(w), F(w))$ such that

$$
\dot{U}(w) \dot{f}(w)=\dot{F}(w), \quad \ddot{U}(w) \geqslant 0 ;
$$

the entropy condition for a physically relevant solution of $(2.2), u \equiv u(x, t)$, requires the following entropy inequality

$$
\frac{\partial}{\partial t} U(u)+\frac{\partial}{\partial x} F(u) \leqslant 0 \quad \text { (weakly) }
$$

to hold for all entropy pairs related through (3.5a). Recalling (3.1b-c), Jensen's inequality and the integral form of $(3.5 \mathrm{~b})$ yield

$$
\begin{aligned}
U\left(v_{\nu-1 / 2}^{-}\right) & \leqslant \frac{1}{\Delta x / 2} \int_{0}^{\Delta x / 2} U\left(u^{R}\left(x / k ; v_{\nu-1}, v_{\nu}\right)\right) d x \\
& \leqslant U\left(v_{\nu}\right)-2 \lambda\left(F\left(v_{\nu}\right)-F_{\nu-1 / 2}^{G}\right), \\
U\left(v_{\nu+1 / 2}^{+}\right) & \leqslant \frac{1}{\Delta x / 2} \int_{-\Delta x / 2}^{0} U\left(u^{R}\left(x / k ; v_{\nu}, v_{\nu+1}\right)\right) d x \\
& \leqslant U\left(v_{\nu}\right)+2 \lambda\left(F\left(v_{\nu}\right)-F_{\nu+1 / 2}^{G}\right),
\end{aligned}
$$


where

$$
F_{\nu+1 / 2}^{G} \equiv F^{G}\left(v_{\nu}, v_{\nu+1}\right)=F\left(u^{R}\left(0^{+} ; v_{\nu}, v_{\nu+1}\right)\right)
$$

is Godunov's numerical entropy flux, consistent with the differential one, $F^{G}(w, w)$ $=F(w)$. Averaging $(3.6 \mathrm{a}-\mathrm{b})$ we find on account of (3.1a) that Godunov's scheme is consistent with the differential entropy inequality (3.5b),

$$
U\left(v_{\nu}(t+k)\right) \leqslant \frac{U\left(v_{\nu-1 / 2}^{-}\right)+U\left(v_{\nu+1 / 2}^{+}\right)}{2} \leqslant U\left(v_{\nu}\right)-\lambda\left(F_{\nu+1 / 2}^{G}-F_{\nu-1 / 2}^{G}\right) .
$$

We now summarize what we have shown in the following

LEMMA 3.1. Assume the CFL condition

$$
\lambda \cdot \max _{u}|a(u)| \leqslant \frac{1}{2}
$$

holds. For $v_{\nu \pm 1 / 2}^{ \pm}$given by

$$
\begin{aligned}
& v_{\nu-1 / 2}^{-}=v_{\nu}-\lambda\left(f_{\nu}-f_{\nu-1}\right)-Q_{\nu-1 / 2}^{G} \Delta v_{\nu-1 / 2}, \\
& v_{\nu+1 / 2}^{+}=v_{\nu}-\lambda\left(f_{\nu+1}-f_{\nu}\right)+Q_{\nu+1 / 2}^{G} \Delta v_{\nu+1 / 2},
\end{aligned}
$$

we have the following entropy inequalities

$$
\begin{aligned}
& U\left(v_{\nu-1 / 2}^{-}\right) \leqslant U\left(v_{\nu}\right)-2 \lambda\left(F\left(v_{\nu}\right)-F_{\nu-1 / 2}^{G}\right), \\
& U\left(v_{\nu+1 / 2}^{+}\right) \leqslant U\left(v_{\nu}\right)+2 \lambda\left(F\left(v_{\nu}\right)-F_{\nu+1 / 2}^{G}\right) .
\end{aligned}
$$

Proof. Inserting the definition of the numerical viscosity coefficient in (2.5), one obtains $(3.10 a-b)$ from $(3.2 b-c)$. The conclusion appears in $(3.6 a-b)$.

Remark. We have shown that Godunov's scheme satisfies the entropy inequality (3.8) by averaging $(3.11 \mathrm{a}-\mathrm{b})$, while assuming the CFL condition (3.2a), $\lambda$. $\max _{u}|a(u)| \leqslant \frac{1}{2}$; the latter was required in order to guarantee that waves issued from the two opposite faces of the $v_{\nu}$-cell do not interact. In the scalar case, an entropy solution is known to exist whether or not these waves interact. Hence, (3.8) follows from the integral form of $(3.5 \mathrm{~b})$ applied over the whole $v_{\nu}$-cell (rather than as we have done over its left and right halves), provided the relaxed CFL condition $\lambda \cdot \max _{u}|a(u)| \leqslant 1$ holds, thus preventing these waves from reaching the cell's other faces. The reason for our introduction of $v_{\nu}(t+k)$ as the average of $v_{\nu-1 / 2}^{-}$and $v_{\nu+1 / 2}^{+}$, each of which satisfies the entropy inequality $(3.11 \mathrm{a}-\mathrm{b})$, will prove itself essential, however, in studying $E$ schemes in Section 5 below. We note that the so introduced averaging is nothing else but a restatement of the following identity, whose verification is left to the reader

$$
H^{G}\left(v_{\nu-1}, v_{\nu}, v_{\nu+1} ; \lambda\right) \equiv \frac{H^{G}\left(v_{\nu-1}, v_{\nu}, v_{\nu} ; 2 \lambda\right)+H^{G}\left(v_{\nu}, v_{\nu}, v_{\nu+1} ; 2 \lambda\right)}{2}
$$

In closing this section, we would like to point out the following geometric interpretation of the numerical viscosity coefficient associated with Godunov's scheme, $Q_{\nu+1 / 2}^{G}$ : integrating (2.2) over the left half of the $v_{\nu+1}$-cell, see Figure 3-1, we find

$$
v_{\nu+1 / 2}^{-}=v_{\nu+1}-2 \lambda\left(f_{\nu+1}-h_{\nu+1 / 2}^{G}\right)
$$


while integration over the right half of the $v_{\nu}$-cell yields, as before,

$$
v_{\nu+1 / 2}^{+}=v_{\nu}+2 \lambda\left(f_{\nu}-h_{\nu+1 / 2}^{G}\right) \text {. }
$$

Subtracting the second from the first, we have

$$
\begin{aligned}
v_{\nu+1 / 2}^{-}-v_{\nu+1 / 2}^{+} & =v_{\nu+1}-v_{\nu}-2 \lambda\left(f_{\nu}+f_{\nu+1}-2 h_{\nu+1 / 2}^{G}\right) \\
& \equiv\left(1-2 Q_{\nu+1 / 2}^{G}\right) \cdot\left(v_{\nu+1}-v_{\nu}\right) .
\end{aligned}
$$

Thus, $1-2 Q_{\nu+1 / 2}^{G}$ gives us the compression ratio $\left(v_{\nu+1 / 2}^{-}-v_{\nu+1 / 2}^{+}\right) /\left(v_{\nu+1}-v_{\nu}\right)$.

4. Lax-Friedrichs Scheme and Its Entropy Satisfying Modification. The LaxFriedrichs scheme [2], [6], given by

$$
\begin{aligned}
v_{\nu}(t+k)= & H^{\mathrm{LF}}\left(v_{\nu-1}, v_{\nu}, v_{\nu+1} ; \lambda\right) \equiv \frac{v_{\nu+1}(t)+v_{\nu-1}(t)}{2} \\
& -\frac{\lambda}{2}\left(f\left(v_{\nu+1}\right)-f\left(v_{\nu-1}\right)\right),
\end{aligned}
$$

has the most allowable numerical viscosity under the total variation nonincreasing requirement (2.8), $Q_{\nu+1 / 2}^{\mathrm{LF}} \equiv 1$. A. Harten has observed [private communication] that the scheme coincides with that of Godunov, when the latter is applied over a staggered grid, see Figure 4-1,

$$
v_{\nu}(t+k)=\frac{1}{2 \Delta x} \int_{-\Delta x}^{\Delta x} u^{R}\left(x / k ; v_{\nu-1}, v_{\nu+1}\right) d x \equiv H^{\mathrm{LF}}\left(v_{\nu-1}, v_{\nu}, v_{\nu+1} ; \lambda\right),
$$

provided the CFL condition

$$
\lambda \cdot \max _{u}|a(u)| \leqslant 1
$$

is met.

$$
v_{v}(t+k)
$$

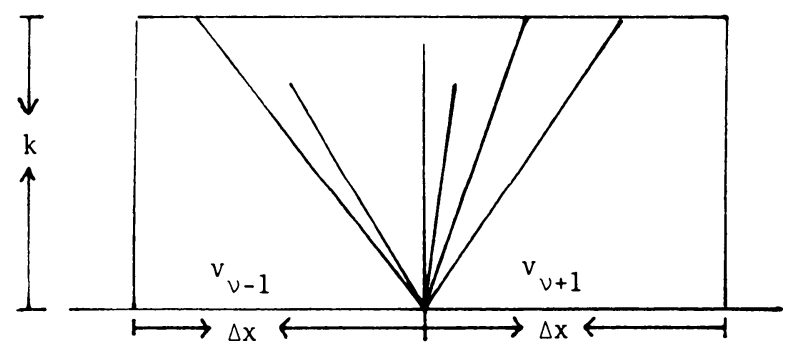

FIGURE 4-1

Integrating the differential entropy inequality (3.5b) over the same domain, we end up with its discrete version

$$
U\left(v_{\nu}(t+k)\right) \leqslant \frac{U\left(v_{\nu+1}(t)\right)+U\left(v_{\nu-1}(t)\right)}{2}-\frac{\lambda}{2}\left(F\left(v_{\nu+1}\right)-F\left(v_{\nu-1}\right)\right)
$$

after little rearrangement, it can be put into the more standard form, compare (3.8),

$$
U\left(v_{\nu}(t+k)\right) \leqslant U\left(v_{\nu}\right)-\lambda\left(F_{\nu+1 / 2}^{\mathrm{LF}}-F_{\nu-1 / 2}^{\mathrm{LF}}\right)
$$

where

$$
L_{\nu+1 / 2}^{\mathrm{LF}} \equiv F^{\mathrm{LF}}\left(v_{\nu}, v_{\nu+1}\right)=\frac{F\left(v_{\nu+1}\right)+F\left(v_{\nu}\right)}{2}-\frac{1}{2 \lambda}\left(U\left(v_{\nu+1}\right)-U\left(v_{\nu}\right)\right)
$$

is LF numerical entropy flux, consistent with the differential one $F^{\mathrm{LF}}(w, w)=F(w)$. 
We note that the LF scheme does not admit a simple averaging of the type introduced above for Godunov's scheme. Instead, one might consider the following modification

$$
\begin{aligned}
v_{\nu}(t+k) & =H^{M}\left(v_{\nu-1}, v_{\nu}, v_{\nu+1} ; \lambda\right) \\
& \equiv \frac{v_{\nu+1}+2 v_{\nu}+v_{\nu-1}}{4}-\frac{\lambda}{2}\left(f\left(v_{\nu+1}\right)-f\left(v_{\nu-1}\right)\right) .
\end{aligned}
$$

The so modified scheme has half the numerical viscosity of the LF-scheme, $Q_{\nu+1 / 2}^{M} \equiv$ $\frac{1}{2}$, and can be rewritten in the desired averaged form

$$
v_{\nu}(t+k)=H^{M}\left(v_{\nu-1}, v_{\nu}, v_{\nu+1}\right) \equiv \frac{v_{\nu-1 / 2}^{-}+v_{\nu+1 / 2}^{+}}{2}
$$

where

$$
\begin{aligned}
& v_{\nu+1 / 2}^{-} \equiv v^{-}\left(v_{\nu-1}, v_{\nu}\right)=\frac{v_{\nu}+v_{\nu-1}}{2}-\lambda\left(f_{\nu}-f_{\nu-1}\right), \\
& v_{\nu+1 / 2}^{+} \equiv v^{+}\left(v_{\nu}, v_{\nu+1}\right)=\frac{v_{\nu+1}+v_{\nu}}{2}-\lambda\left(f_{\nu+1}-f_{\nu}\right) .
\end{aligned}
$$

The new scheme introduced, (4.4), can also be viewed as a two-cell averaging of two noninteracting Riemann problems, see Figure 3-1,

$$
\begin{aligned}
v_{\nu}(t+k) & =H^{M}\left(v_{\nu-1}, v_{\nu}, v_{\nu+1}\right) \\
& \equiv \frac{1}{2 \Delta x}\left[\int_{-\Delta x / 2}^{\Delta x / 2} u^{R}\left(x / k ; v_{\nu-1}, v_{\nu}\right) d x+\int_{-\Delta x / 2}^{\Delta x / 2} u^{R}\left(x / k ; v_{\nu}, v_{\nu+1}\right) d x\right]
\end{aligned}
$$

provided the CFL limitation

$$
\lambda \cdot \max _{u}|a(u)| \leqslant \frac{1}{2}
$$

is met. Integrating the entropy inequality (3.5b) over the same two-cell domain, the scheme is found to satisfy that entropy inequality in its standard discrete version

$$
U\left(v_{\nu}(t+k)\right) \leqslant U\left(v_{\nu}(t)\right)-\lambda\left(F_{\nu+1 / 2}^{M}-F_{\nu-1 / 2}^{M}\right)
$$

with a numerical entropy flux

$$
F_{\nu+1 / 2}^{M} \equiv F^{M}\left(v_{\nu}, v_{\nu+1}\right)=\frac{F\left(v_{\nu+1}\right)+F\left(v_{\nu}\right)}{2}-\frac{1}{4 \lambda}\left(U\left(v_{\nu+1}\right)-U\left(v_{\nu}\right)\right)
$$

consistent with the differential one, $F^{M}(w, w)=F(w)$.

In analogy with Lemma 3 , we are now ready to state

\section{LEMMA 4.1. Assume the CFL condition}

$$
\lambda \cdot \max _{u}|a(u)| \leqslant \frac{1}{2}
$$

holds. For $v_{\nu_{ \pm 1 / 2}^{ \pm}}^{ \pm}$, given by

$$
\begin{aligned}
& v_{\nu-1 / 2}^{-}=v_{\nu}-\lambda\left(f_{\nu}-f_{\nu-1}\right)-\frac{1}{2} \Delta v_{\nu-1 / 2}, \\
& v_{\nu+1 / 2}^{+}=v_{\nu}-\lambda\left(f_{\nu+1}-f_{\nu}\right)+\frac{1}{2} \Delta v_{\nu+1 / 2},
\end{aligned}
$$

we have the following entropy inequalities:

$$
U\left(v_{\nu-1 / 2}^{-}\right) \leqslant U\left(v_{\nu}\right)-2 \lambda\left(F\left(v_{\nu}\right)-F_{\nu-1 / 2}^{M}\right),
$$




$$
U\left(v_{\nu+1 / 2}^{+}\right) \leqslant U\left(v_{v}\right)+2 \lambda\left(F\left(v_{v}\right)-F_{\nu+1 / 2}^{M}\right) .
$$

Proof. The RHS of (4.8a) and (4.8b) coincide with $H^{\mathrm{LF}}\left(v_{\nu-1}, v_{\nu}, v_{\nu} ; 2 \lambda\right)$ and $H^{\mathrm{LF}}\left(v_{\nu}, v_{\nu+1}, v_{\nu+1} \cdot 2 \lambda\right)$, respectively; applying for the latter the LF entropy inequality as quoted in $(2.3 \mathrm{a}-\mathrm{b})$, one obtains the conclusion $(4.9 \mathrm{a}-\mathrm{b})$.

Remark. In [7], P. D. Lax gave a direct proof of the entropy inequality (4.3), for the LF scheme approximating an arbitrary system of conservation laws. (In comparison, the arguments used in the above scalar analysis requires the existence of an entropy satisfying the Riemann solution in the large.) Since the modified scheme is nothing else but an average of two LF-solvers, Lax's result goes over in this case; that is, for an arbitrary system of conservation laws, both LF and the modified scheme, satisfy the entropy inequality for all entropy pairs associated with the differential system. ${ }^{\dagger}$ As much as we are aware, these are the only two known examples satisfying the entropy condition in such generality.

5. The Entropy Condition and $E$ Schemes. In this section we study difference schemes containing no less numerical viscosity than that of Godunov, $Q \geqslant Q^{G}$. Such $E$ schemes-after Osher [12]-are shown to converge to the unique physically relevant solution of (2.2), provided the CFL limitation

$$
\lambda\left|\left(f\left(v_{\nu}\right)-h_{\nu+1 / 2}\right)+\left(f\left(v_{\nu+1}\right)-h_{\nu+1 / 2}\right)\right| \leqslant \frac{1}{2}\left|v_{\nu+1}-v_{\nu}\right|
$$

is met. Ideally, one would like to allow the relaxed CFL limitation (2.10) to be used; the reason for introducing the stricter (5.1) (half the usual CFL number) stems from the fact that we were unable to rewrite the LF scheme in the desirable averaged form as discussed in Section 4. We note that (5.1) takes the equivalent form

$$
\left|Q_{\nu+1 / 2}\right| \leqslant \frac{1}{2} \text {, }
$$

which, in the case of Godunov's scheme, amounts to preventing waves interaction. As before, such a CFL limitation yields, in particular, the consistency relation (2.11), characterizing essentially 3 -point schemes.

THEOREM 5.1. An E scheme converges to the physically relevant solution of (2.2), under the CFL restriction (5.1).

Proof. Convergence was established in Lemma 2.1 and Lemma 2.2, since an $E$ scheme is necessarily total variation and maximum norm nonincreasing (e.g., $[10$, Section 2])

$$
\lambda\left|\frac{\Delta f_{\nu+1 / 2}}{\Delta v_{\nu+1 / 2}}\right| \leqslant Q_{\nu+1 / 2}^{G} \leqslant Q_{\nu+1 / 2} \leqslant \frac{1}{2}<1 .
$$

We turn to examine the entropy inequality. We attach the superscript $G, M$, and $E$ to distinguish between Godunov's scheme (3.1), the modified scheme (4.4), and the $E$ scheme under consideration, (2.7),

$$
v_{\nu}(t+k)=v_{\nu}(t)-\frac{\lambda}{2}\left(f\left(v_{\nu+1}(t)\right)-f\left(v_{\nu-1}(t)\right)\right)+\frac{1}{2}\left(\Delta\left(Q_{\nu-1 / 2} \Delta v_{\nu-1 / 2}(t)\right)\right) .
$$

We rewrite the latter in the averaged form

$$
v_{\nu}(t+k)=\frac{v_{\nu-1 / 2}^{E-}+v_{\nu+1 / 2}^{E+}}{2},
$$

\footnotetext{
${ }^{\dagger}$ For the exact CFL limitation in this case, see [7].
} 
where

$$
\begin{aligned}
& v_{\nu-1 / 2}^{E-}=v_{\nu}-\lambda\left(f_{\nu}-f_{\nu-1}\right)-Q_{\nu-1 / 2} \Delta v_{\nu-1 / 2}, \\
& v_{\nu+1 / 2}^{E+}=v_{\nu}-\lambda\left(f_{\nu+1}-f_{\nu}\right)+Q_{\nu+1 / 2} \Delta v_{\nu+1 / 2} .
\end{aligned}
$$

Recall the corresponding averaging forms for Godunov's scheme, see (3.10),

$$
\begin{aligned}
& v_{\nu-1 / 2}^{G-}=v_{\nu}-\lambda\left(f_{\nu}-f_{\nu-1}\right)-Q_{\nu-1 / 2}^{G} \Delta v_{\nu-1 / 2}, \\
& v_{\nu+1 / 2}^{G+}=v_{\nu}-\lambda\left(f_{\nu+1}-f_{\nu}\right)+Q_{\nu+1 / 2}^{G} \Delta v_{\nu+1 / 2},
\end{aligned}
$$

and that for the modified scheme, see (4.8),

$$
\begin{aligned}
& v_{\nu-1 / 2}^{M-}=v_{\nu}-\lambda\left(f_{\nu}-f_{\nu-1}\right)-\frac{1}{2} \Delta v_{\nu-1 / 2}, \\
& v_{\nu+1 / 2}^{M+}=v_{\nu}-\lambda\left(f_{\nu+1}-f_{\nu}\right)+\frac{1}{2} \Delta v_{\nu+1 / 2} .
\end{aligned}
$$

According to our assumption

$$
Q_{\nu \pm 1 / 2}=\theta_{\nu \pm 1 / 2} Q_{\nu \pm 1 / 2}^{G}+\left(1-\theta_{\nu \pm 1 / 2}\right) \frac{1}{2}, \quad 0 \leqslant \theta_{\nu \pm 1 / 2} \leqslant 1 .
$$

Multiply (5.4a) by $\theta_{\nu-1 / 2},(5.5 a)$ by $\left(1-\theta_{\nu-1 / 2}\right)$ and add to find that (5.3a) amounts to

$$
v_{\nu-1 / 2}^{E-}=\theta_{\nu-1 / 2} v_{\nu-1 / 2}^{G-}+\left(1-\theta_{\nu-1 / 2}\right) v_{\nu-1 / 2}^{M-}
$$

similarly, multiplying $(5.4 \mathrm{~b})$ by $\theta_{\nu+1 / 2},(5.5 \mathrm{~b})$ by $\left(1-\theta_{\nu+1 / 2}\right)$ and adding, we end up with (5.3b) having the form

$$
v_{\nu+1 / 2}^{E+}=\theta_{\nu+1 / 2} v_{\nu+1 / 2}^{G+}+\left(1-\theta_{\nu+1 / 2}\right) v_{\nu+1 / 2}^{M+} .
$$

Averaging the last two equalities, (5.2) becomes

$$
\begin{aligned}
v_{\nu}(t+k)= & \frac{\theta_{\nu-1 / 2}}{2} v_{\nu-1 / 2}^{G-}+\frac{\left(1-\theta_{\nu-1 / 2}\right)}{2} v_{\nu-1 / 2}^{M-} \\
& +\frac{\theta_{\nu+1 / 2}}{2} v_{\nu+1 / 2}^{G+}+\frac{\left(1-\theta_{\nu+1 / 2}\right)}{2} v_{\nu+1 / 2}^{M+} .
\end{aligned}
$$

Thus, we see that every $E$ scheme can be written as a convex combination of one-sided averaged Riemann solutions.

Let $(U(w), F(w))$ be an entropy pair associated with (2.2). By the convexity of $U$, (5.7) implies

$$
\begin{aligned}
U\left(v_{\nu}(t+k)\right) \leqslant & \frac{\theta_{\nu-1 / 2}}{2} U\left(v_{\nu-1 / 2}^{G-}\right)+\frac{\left(1-\theta_{\nu-1 / 2}\right)}{2} U\left(v_{\nu-1 / 2}^{M-}\right) \\
& +\frac{\theta_{\nu+1 / 2}}{2} U\left(v_{\nu+1 / 2}^{G+}\right)+\frac{\left(1-\theta_{\nu+1 / 2}\right)}{2} U\left(v_{\nu+1 / 2}^{M+}\right) .
\end{aligned}
$$

Next, we invoke the entropy inequalities concluded in Lemmas 3.1 and 4.1

$$
\begin{aligned}
& U\left(v_{\nu-1 / 2}^{G-}\right) \leqslant U\left(v_{\nu}\right)-2 \lambda\left(F\left(v_{\nu}\right)-F_{\nu-1 / 2}^{G}\right), \\
& U\left(v_{\nu+1 / 2}^{G+}\right) \leqslant U\left(v_{\nu}\right)+2 \lambda\left(F\left(u_{\nu}\right)-F_{\nu+1 / 2}^{G}\right), \\
& U\left(v_{\nu-1 / 2}^{M-}\right) \leqslant U\left(v_{\nu}\right)-2 \lambda\left(F\left(v_{\nu}\right)-F_{\nu-1 / 2}^{M}\right), \\
& U\left(v_{\nu+1 / 2}^{M+}\right) \leqslant U\left(v_{\nu}\right)+2 \lambda\left(F\left(v_{\nu}\right)-F_{\nu+1 / 2}^{M}\right) .
\end{aligned}
$$


When these are inserted into (5.8), we end up with the desired entropy inequality

$$
U\left(v_{\nu}(t+k)\right) \leqslant U\left(v_{\nu}\right)-\lambda\left(F_{\nu+1 / 2}^{E}-F_{\nu-1 / 2}^{E}\right)
$$

with a numerical entropy flux

$$
F_{\nu+1 / 2}^{E}=\theta_{\nu+1 / 2} F_{\nu+1 / 2}^{G}+\left(1-\theta_{\nu+1 / 2}\right) F_{\nu+1 / 2}^{M}
$$

consistent with the differential one, $F^{E}(\cdots, w, w, \cdots)=F(w)$.

Remarks. (i). An explicit formula for Godunov's numerical flux,

$$
h^{G}\left(v_{\nu}, v_{\nu+1}\right)=\operatorname{Min}_{v}\left[\operatorname{sgn}\left(v_{\nu+1}-v_{\nu}\right) f(v)\right], \quad v_{\nu+1 / 2}^{\min } \leqslant v \leqslant v_{\nu+1 / 2}^{\max },
$$

was given in [12]; here $v_{\nu+1 / 2}^{\min / \max }=\operatorname{Min} / \operatorname{Max}\left(v_{\nu}, v_{\nu+1}\right)$. Hence, an equivalent characterization for $E$ schemes, requiring

$$
\operatorname{sgn}\left(v_{\nu+1}-v_{\nu}\right)\left[h_{\nu+1 / 2}-f(v)\right] \leqslant 0, \quad v_{\nu+1 / 2}^{\min } \leqslant v \leqslant v_{\nu+1 / 2}^{\max },
$$

shows that a 3-point monotone scheme is an $E$ scheme. Unfortunately, $E$ schemes, like monotone ones, are at most first-order accurate [12].

(ii) We have seen that $E$ schemes satisfy the entropy inequality (5.9) for all entropy functions, $U(\cdot)$; their corresponding numerical entropy fluxes are given as convex combinations of two numerical fluxes associated with monotone schemes-Godunov and the modified LF scheme (4.4). Hence, an $L^{1}$-convergence rate estimate of order $(\Delta x)^{1 / 2}$ follows along the lines of [9, Theorem IV]

$$
\|v(\cdot, t)-u(\cdot, t)\|_{L^{1}} \leqslant\|v(\cdot, t=0)-u(\cdot, t=0)\|_{L^{1}}+K \cdot(t \Delta x)^{1 / 2} .
$$

Considerations of the constant coefficients case shows this $L^{1}$-estimate to be sharp, e.g., [11, Sections 9 and 10].

(iii) As an immediate corollary from Theorem 5.1 we obtain verification of the following "folklore" result.

COROLlaRY 5.2. A conservative difference scheme with a nonvanishing numerical viscosity, $0<Q_{\min } \leqslant Q_{\nu+1 / 2}(\lambda) \leqslant \frac{1}{2}$, is converging to the unique entropy solution for sufficiently small mesh ratio, $\lambda$.

Such nonvanishing viscosity schemes were specifically "tailored", for example, in [5, Section 5]. Here we note, that the CFL-like restriction on the mesh ratio, $\lambda$, depends heavily on the behavior of the flux, $f$, near the sonic points.

6. Numerical Viscosity and the Entropy Condition. In this section we would like to systematize the kind of arguments introduced above, emphasizing those essential ingredients which prevail in the more general context.

We consider a general conservative scheme which we rewrite in the averaged form, compare (2.7),

$$
\begin{aligned}
& v_{\nu}(t+k) \\
& =\frac{\left[v_{\nu}(t)-\lambda\left(f_{\nu}-f_{\nu-1}\right)-Q_{\nu-1 / 2} \Delta v_{\nu-1 / 2}\right]+\left[v_{\nu}(t)-\lambda\left(f_{\nu+1}-f_{\nu}\right)+Q_{\nu+1 / 2} \Delta v_{\nu+1 / 2}\right]}{2} ;
\end{aligned}
$$

the entropy condition follows by constructing a consistent discrete entropy inequality for each of the averaged terms on the RHS (6.1), thus opening the door for 
showing the former by means of comparison. For that purpose, pick a 3-point entropy condition satisfying the scheme

$$
\mathbf{v}_{\nu}(t+k)=\mathbf{H}\left(v_{\nu-1}, v_{\nu}, v_{\nu+1} ; f, \lambda\right) \equiv v_{\nu}-\lambda\left(\mathbf{h}_{\nu+1 / 2}-\mathbf{h}_{\nu-1 / 2}\right)
$$

such that the following holds:

Assumption. The numerical flux $\mathbf{h}_{\nu+1 / 2}$ is independent of the mesh ratio $\lambda$.

The plausibility of the above assumption stems from the fact that the Riemann problem admits similarity solution $u^{R}\left(x, t ; u_{\text {left }}, u_{\text {right }}\right) \equiv u^{R}\left(x / t ; u_{\text {left }}, u_{\text {right }}\right)$, and hence all difference approximations based on Riemann solvers must satisfy such a requirement; this is not the case, for example, with the LF scheme (4.1), where we were forced to consider instead its modification (4.4).

The reason for introducing the last assumption is becoming clear upon writing

$$
\begin{aligned}
\mathbf{H}\left(v_{\nu-1}, v_{\nu}, v_{\nu+1} ; \lambda\right) & \\
\quad= & \frac{\left[v_{\nu}-\lambda\left(f_{\nu}-f_{\nu-1}\right)-\mathbf{Q}_{v-1 / 2} \Delta v_{v-1 / 2}\right]+\left[v_{v}-\lambda\left(f_{v+1}-f_{v}\right)+\mathbf{Q}_{v+1 / 2} \Delta v_{v+1 / 2}\right]}{2},
\end{aligned}
$$

where, see (2.5),

$$
\mathbf{Q}_{\nu+1 / 2} \equiv \lambda \frac{f_{\nu}+f_{\nu+1}-2 \mathbf{h}_{\nu+1 / 2}}{\Delta v_{\nu+1 / 2}}
$$

depends linearly on $\lambda$; hence, the two averaged terms on the RHS of (6.3)-abbreviated as before by $\mathbf{v}_{\nu-1 / 2}^{-}$and $\mathbf{v}_{\nu+1 / 2}^{+}$-can be equivalently expressed as

$$
\mathbf{v}_{\nu-1 / 2}^{-} \equiv \mathbf{H}\left(v_{\nu-1}, v_{\nu}, v_{\nu} ; 2 \lambda\right), \quad \mathbf{v}_{\nu+1 / 2}^{+} \equiv \mathbf{H}\left(v_{\nu}, v_{\nu}, v_{\nu+1} ; 2 \lambda\right),
$$

each of which satisfies the entropy inequality, provided the CFL limitation is being halved. Termwise comparison of the averaged forms, (6.1) and (6.3), shows their difference only in the numerical viscosity coefficients; assuming $Q_{\nu+1 / 2}$ to vary between two coefficients of numerical viscosity associated with entropy satisfying schemes, we are able to represent (6.1) as a convex combination of the latter. The discrete entropy inequality follows for the corresponding convex combination of entropy fluxes.

We have shown

THEOREM 6.1. Consider the difference scheme (6.1) and assume that the CFL condition

$$
\left|Q_{\nu+1 / 2}\right| \equiv \lambda\left|\frac{f_{\nu}+f_{\nu+1}-2 h_{\nu+1 / 2}}{\Delta v_{\nu+1 / 2}}\right| \leqslant \frac{1}{2}
$$

holds. ${ }^{\dagger \dagger}$ Then, the scheme satisfies the entropy condition, provided we can find another entropy satisfying difference approximation with less numerical dissipation, $\mathbf{Q}_{\nu+1 / 2}$,

$$
\mathbf{Q}_{\nu+1 / 2} \leqslant Q_{\nu+1 / 2} \text {. }
$$

\footnotetext{
${ }^{\dagger \dagger}$ One may assume, instead, $\left|Q_{v+1 / 2}\right| \leqslant\left|\check{Q}_{v+1 / 2}\right|, \check{Q}_{v+1 / 2}$ denoting the numerical viscosity coefficient of a difference scheme admitting the desired entropy satisfying averaged form.
} 
The corresponding numerical entropy flux is given by

$$
F_{\nu+1 / 2}=\left[\frac{\frac{1}{2}-Q_{\nu+1 / 2}}{\frac{1}{2}-\mathbf{Q}_{\nu+1 / 2}}\right] \cdot \mathbf{F}_{\nu+1 / 2}+\left[\frac{Q_{\nu+1 / 2}-\mathbf{Q}_{\nu+1 / 2}}{\frac{1}{2}-\mathbf{Q}_{\nu+1 / 2}}\right] \cdot F_{\nu+1 / 2}^{M} .
$$

ICASE

NASA Langley Research Center

Hampton, Virginia 23665

1. M. CRANDall \& A. MAJDa, "Monotone difference approximations for scalar conservation laws," Math. Comp., v. 34, 1980, pp. 1-21.

2. K. O. Friedrichs, "Symmetric hyperbolic linear differential equations," Comm. Pure Appl. Math., v. 7,1954, pp. $345-392$

3. J. GLImM, "Solution in the large for nonlinear hyperbolic systems of equations," Comm. Pure Appl. Math., v. 18, 1965, pp. 697-715.

4. S. K. Godunov, "A finite difference method for the numerical computation of discontinuous solutions of the equations of flow dynamics," Mat. Sh., v. 47, 1959, pp. 271-290.

5. A. HaRTen, "High resolution schemes for hyperbolic conservation laws," J. Comput. Phys., v. 49, 1983, pp. 357-393.

6. P. D. LAX, "Weak solutions of nonlinear hyperbolic equations and their numerical computation," Comm. Pure Appl. Math., v. 7, 1954, pp. 159-193.

7. P. D. LAX, "Shock waves and entropy," in Contributions to Nonlinear Functional Analysis (E. A. Zarantonello, ed.), Academic Press, New York, 1971, pp. 603-634.

8. A. MAJDa \& S. Osher, "Numerical viscosity and the entropy condition," Comm. Pure Appl. Math., v. 32,1979 , pp. $797-838$.

9. R. SANDERS, "On convergence of monotone finite difference schemes with variable spatial efficiency," Math. Comp., v. 40, 1983, pp. 91-106.

10. E. TADMOR, "The large-time behavior of the scalar, genuinely nonlinear Lax-Friedrichs scheme," Math. Comp., this issue.

11. V. THOMEE, "Stability theory for partial difference operators," SIAM Rev., v. 11, 1969, pp. 152-195.

12. S. OSHER, "Riemann solvers, the entropy condition and difference approximations," SIAM J. Numer. Anal., v. 21, 1984, pp. 217-235. 